\title{
CAPACITY MANAGEMENT AND PATIENT SCHEDULING IN AN OUTPATIENT CLINIC USING DISCRETE EVENT SIMULATION
}

\author{
Gokce Akin \\ Graduate Program in Operations Research \\ North Carolina State University \\ Raleigh, NC 27695, USA
}

Julie S. Ivy

Edward P. Fitts Department of Industrial
and Systems Engineering
North Carolina State University
Raleigh, NC 27695, USA

\author{
Todd R. Huschka \\ Thomas R. Rohleder \\ Yariv N. Marmor \\ Department of Health Care Policy and Research \\ Mayo Clinic \\ 200 1st ST SW \\ Rochester, MN 55905, USA
}

\begin{abstract}
Capacity management and scheduling decisions are important for managing an outpatient clinic in which multiple classes of patients are treated. After an appointment is scheduled, it can be rescheduled, cancelled, or a patient may not show-up on their appointment day. This study simulates the behavior of patients with regard to the time to appointment, examining different demand rates and service times for each patient class (new external patients, internal patients, established patients and subsequent visit patients); we also consider different delay-dependent reschedule, cancellation, and no-show rates. A discrete event simulation model is developed to analyze the effects of allowing different appointment windows, i.e., the maximum time between the appointment request date and the actual appointment date, for different patient classes. Capacity utilization, patient access, and financial rewards are used as the performance indicators.
\end{abstract}

\section{INTRODUCTION}

In an outpatient clinic, which observes several appointment requests from different patients classes every day, it is essential to provide timely access to the patients although their conditions may not be critical, while preventing overbooked or idle slots in the physician calendars. Due to limited capacity in the clinic, it is important to have efficient capacity management and patient scheduling strategies.

There are several uncertainties associated with the patients. In the outpatient clinic of concern in this paper, the demand is observed from multiple patient classes. Namely the patient classes are internal patients who are referred to this department by other departments in the clinic, new external patients who are new to the clinic, established patients who were seen previously in this clinic, and subsequent visit patients who are receiving follow-up care from a previous visit as any of the other three types of patients. An important patient characteristic is that they have different tendencies for canceling, rescheduling, or not showing up for their appointments. Galluci, Swartz, and Hackerman (2005) indicated that the cancel- 
lation and no-show rates increase with the appointment delay, which is defined as the actual time between the appointment request date and the assigned appointment date. We also observe the same pattern in our data analysis and in addition to cancellation and no-show rates we also identify that the reschedule rates increase as the delay increases. Further we observe that these behavioral functions have different rates of increase for each patient class. Given that different patient classes have different revenues associated with them, we take the net profit in addition to the seen patient percentages and utilizations into account while evaluating different strategies, because early appointments for a certain patient class may increase the seen patient percentages, however since it may block other patients from entering the clinic in a timely manner, it does not necessarily increase the net profit.

The current traditional scheduling system has distinct calendars for each physician and physicians decide when to accept a patient of which class. These calendars have predefined slots in which only certain patient classes can be assigned. In addition appointments are assigned to these slots if the appointment type and slot description match. If a certain type of slots is full, then the next incoming patient appointment request will be scheduled further into the future. These slot designations and distinct calendars for each physician result in overbooked or idle slots.

In this paper, instead of predefined slots we use generic slots of specified length whose number is set based on the current usable overall capacity for each subspecialty in the clinic. In other words, we use physician pools based on their subspecialties and assume that the patient is willing to see any of the physicians in that pool. This paper is an extension of our previous work Akin et al. (2013), in which we have shown the effects of reducing appointment windows for all patient types at the same time by the same amount, under the performance indicators of the seen patient percentages and utilizations. In that study we observed that reducing all appointment windows improves the system, however we did not measure the effect on the daily net revenue. In that paper we also ignored the fact that the subsequent visits were actually generated by other types of appointments, and we assumed that all appointment types were independent of each other. In this study we consider different appointment windows for different patient classes, different revenues obtained by seeing patients of different classes, and the subsequent visits are generated by the other three classes of patients.

In the next section, the literature review is presented; in Section 3 the data analysis, simulation model and the scenarios are presented, followed by Section 4 in which we explain our findings based on our experimental analysis via simulation. Finally, Section 5 concludes with a summary and final discussion.

\section{LITERATURE REVIEW}

Patient scheduling and capacity management have been explored extensively in the literature. Cayirli and Veral (2003) and Gupta and Denton (2008) have reviewed the most important and recent papers within their surveys. In this section we consider the papers which are close to our problem. We classify these papers into three major groups: single-class patients with single resource, multi-class patients with single resource, and multi-class patients with multiple resources.

Within the single class of patients and single resource group, authors handle a system in which all patients have the same demand rates, rewards and behaviors, and the system has only one resource. The main objective in all of the papers in this classification, except Green and Savin (2008), is to maximize the expected net profit which depends on the seen patient percentages as well as the physician utilization. On the other hand, Green and Savin (2008) find the largest panel size that can be handled by the clinic. In terms of behaviors, LaGanga and Lawrence (2007) consider no-shows, with an assumption the no-show probability was fixed. Green and Savin (2008) and Patrick (2012) considered delay dependent no-show rates. Liu, Ziya, and Kulkarni (2010) and Feldman et al. (2012) considered both delay dependent cancellation and no-show rates.

Papers with multiple classes of patients with a single resource consider a system and different patient classes that have different demand rates and/or different behavior function rates and/or different priorities (or rewards) associated with them. Similar to the previous group of papers, the papers in this class also 
have the objective of maximizing the expected net profit as a function of the seen patients and the physician utilization. Within this class, Patrick, Puterman and Queyranne (2008) and Ayvaz and Huh (2010) do not consider cancellations and no-shows, whereas patient class-based fixed no-show rates are assumed by Cayirli, Veral, and Rosen (2008), Muthuraman and Lawley (2008), Chakraborty, Muthuraman, and Lawley (2010), Zeng et al. (2010), and Ratcliffe, Gilland, and Marucheck (2012). In addition to the class-based fixed no-show rates, Shutz and Kolisch (2013) also considers class-based cancellation rates. Unlike the previously mentioned papers, Samorani and LaGanga (2011) use delaydependent class-based no-show rates but without considering the cancellations.

In the last group of papers which handles a system with multiple classes of patients and multiple resources, there are different characteristics associated with each patient class, and for this category of papers the system has multiple resources which could be utilized. Actually this group can also be divided into two subgroups: The first group handles the systems with multiple resources used during a single patient visit, and the second group handles the systems with a single resource that is chosen from a set of resources based on the appointment type. Within this group, neither Gupta and Wang (2008) nor White, Froehle, and Klassen (2011) considered patient behaviors, whereas delay-based no-show rates are used by Huh, Liu, and Truong (2013).

Among the papers mentioned above, only White, Froehle, and Klassen (2011) used a simulation model to analyze different appointment scheduling and capacity allocation strategies. All other papers used analytical models, like MDP models, queuing models, and mathematical programming models, to obtain the best appointment scheduling policy. Due to the complicated nature of the system considered in this paper, we use simulation to analyze the effects of changing appointment windows for different patient classes in the clinic. An important contribution of this paper is related to reschedules, because in the literature reschedule behavior is typically ignored due to its complexity, although the reschedule rates are not negligible especially in outpatient clinics. Ignoring the effects of reschedules can yield solutions that are not feasible for the clinics in terms of capacity, because with rescheduling the demand is simply moved to other days, thus it still creates load on the system. To the best of our knowledge the previous multi-patient studies do not consider the dependency between the patient classes. Hence, having subsequent visit appointments generated by other appointments is another contribution of this paper to the patient scheduling literature. Finally, our previous basis paper Akin et al. (2013) and this paper are the first to handle a system with multiple patient classes that have different delay-based no-show, reschedule and cancellation probabilities. In summary, the main distinctions of this work compared to Akin et al. (2013) paper are, the dependency of the subsequent visits with the other patient classes, consideration of patient-based revenues and penalties associated with cancellation and reschedules, and the allowance for different appointment windows for different patient classes.

\section{METHODOLOGY}

Two years of data has been used to characterize the current state of the clinic, to find both the patient related attributes (demand and behavioral functions) and capacity related parameters (daily available capacities for each subspecialty). After obtaining the necessary parameters and functions we built a simulation model in Arena, validate the current system model with the actual data, and we experiment with different scenarios to see the effects of changes.

\subsection{Current State and Data Analysis}

Currently in the clinic all patient classes have an appointment window of 12 weeks. In terms of daily demand, we have observed that each patient class has a different appointment request rate. Based on the 2year demand data, the average number of appointment requests per day is 17 for the internal patients, 10 for the new external patients, 36 for the established patients and 44 for the subsequent visit patients. However, in reality the subsequent visits are generated by other patients classes since this type of visit is due to follow up from a previous appointment. For this reason, we analyze the subsequent visit impact 
created by other types of patients. Based on our analysis, an internal patient visit creates 0.55 subsequent visits per day, while this number is 1.12 for the new external patients and 0.65 for the established patients. Thus subsequent visit patients are created as a function of the other three types of patients.

Each of the four patient classes can request an appointment from any of the eight subspecialty. Internal patients are expected to have 40 minute appointments, new external patients have 60 minute appointments, subsequent visit patients have 20 minute appointments regardless of the subspecialty that they need, while established patients can have appointments of length 20,40 , and 60 minutes, with probabilities of $0.077,0.781$, and 0.142 , respectively based on the current data.

In the outpatient clinic that our study is based on, a patient can cancel or reschedule his/her initially assigned appointment before the appointment date, or he/she may not show-up on the assigned appointment date. All of these behaviors depend on the patient class, as shown in Figure 1. Different graphs for each behavior with have four curves representing each patient class. Based on this figure we can conclude that we need to have different functions, which are based on delay (time between the appointment request and the actual appointment) and patient class. The probability curves in Figure 1 are obtained based on a nine week horizon, because the number of appointments that are actually scheduled later than that is very low.

We can easily observe from the graphs that the probability curves for the reschedule and cancellation are significantly higher than the no-show curves and the probabilities increase as the appointment delay increases. This suggests that as the appointment delay increases, more changes are observed in the appointments. Moreover, by looking at the graph with the "seen" curves, we can see that the probability of being seen for the new external patients reduces drastically as we increase the appointment delay. This can be explained by the fact that the new external patients are new to the clinic, and since they do not have a commitment yet, it is more likely for this class to cancel, i.e., if these patients have large delays in their appointments they will not be seen. This reduction is also significant for the internal patients compared to the subsequent visit patients and established patients, because the internal patients are referred to this department by other departments and although they are already in the clinic they are "new" to this department, so if they cannot be admitted by the department as soon as possible, their probability of seen decreases significantly. Additionally, based on the data we know that $17 \%$ of the appointments are rescheduled in this clinic, either to a later date or an earlier date. Hence, this movement of appointments between days has to be taken into account in order to have feasible strategies.

We assume that each seen internal patient yields revenue of 0.8 units, a new external patient yields 3 units, a subsequent visit patient yields 2 units, and an established patient yields 1 unit, as a function of the relative value unit. Moreover, we set the penalties for cancellations and rescheduling based on the effort that the appointment scheduler spends. We assume that cancelling or rescheduling an appointment has a penalty of 0.05 units based on an expert opinion from the clinic.

The final part of our data analysis is associated with the capacity, which was the most challenging component of this study, because in the clinic there are several physician-based calendars in which there are several slot types. Using these calendars we need to capture the available capacity for patient visits. For this reason, using several data files and with expert opinion we identify the available hours of slots in the current scheduling system. Note that these calendars are grouped into eight subspecialties, which we will be using as our resource sets, each of which has its own overall capacity. At this point, we can say that in this setting we have multiple resources that act as multiple single resources because they are grouped into independent subspecialties.

\subsection{Simulation Model}

We develop a simulation model, whose flow chart is shown in Figure 2, in Arena in order to conduct an experimental study based on several scenarios. Note that the scenarios are explained in the next subsection. In this simulation model, internal, new external and established patients arrive based on their daily appointment request rates, while subsequent patient visits are generated from these appointment types as 
Akin, Ivy, Huschka, Rohleder, and Marmor

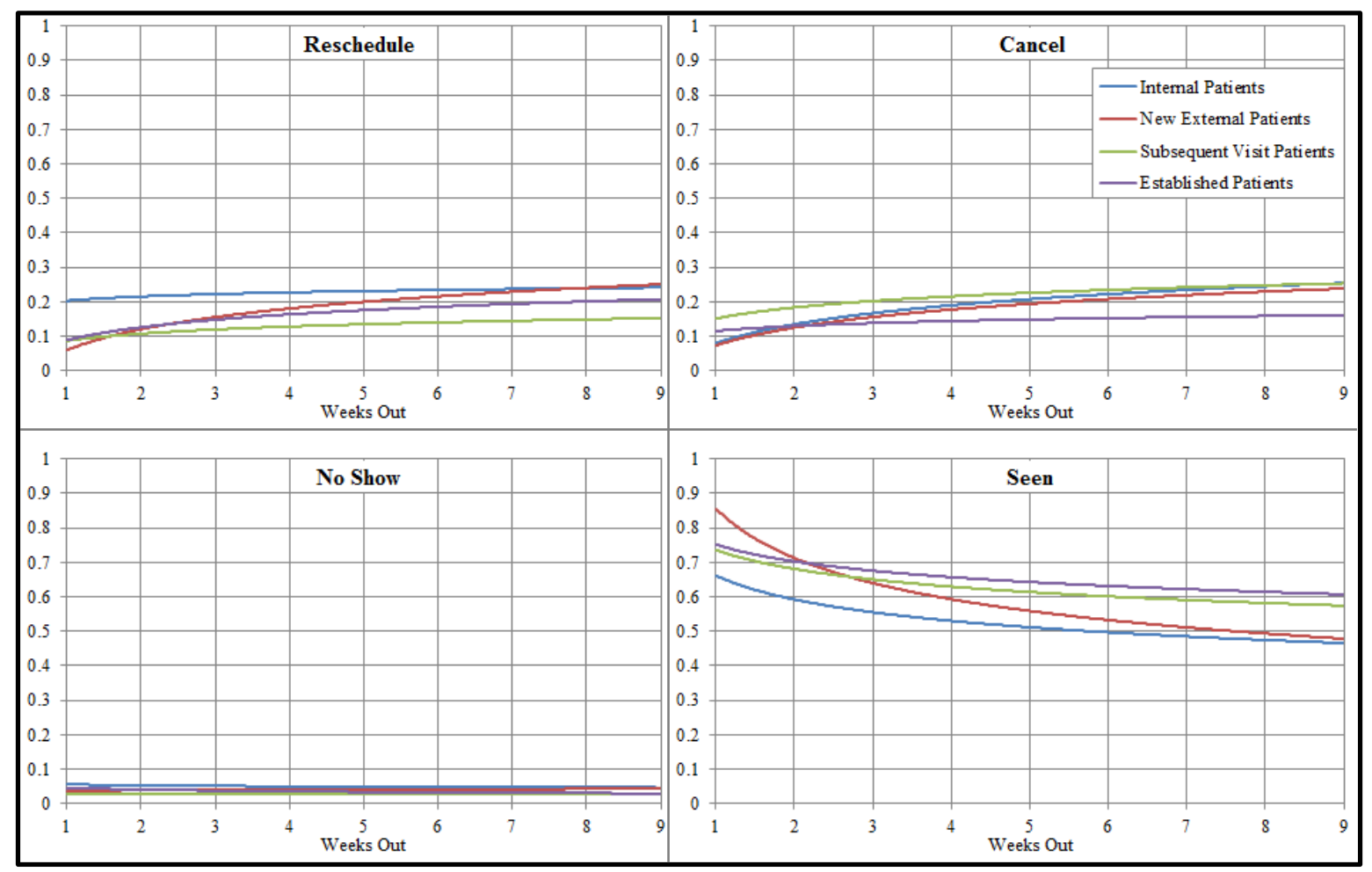

Figure 1: Probability curves to represent each behavior for each patient type

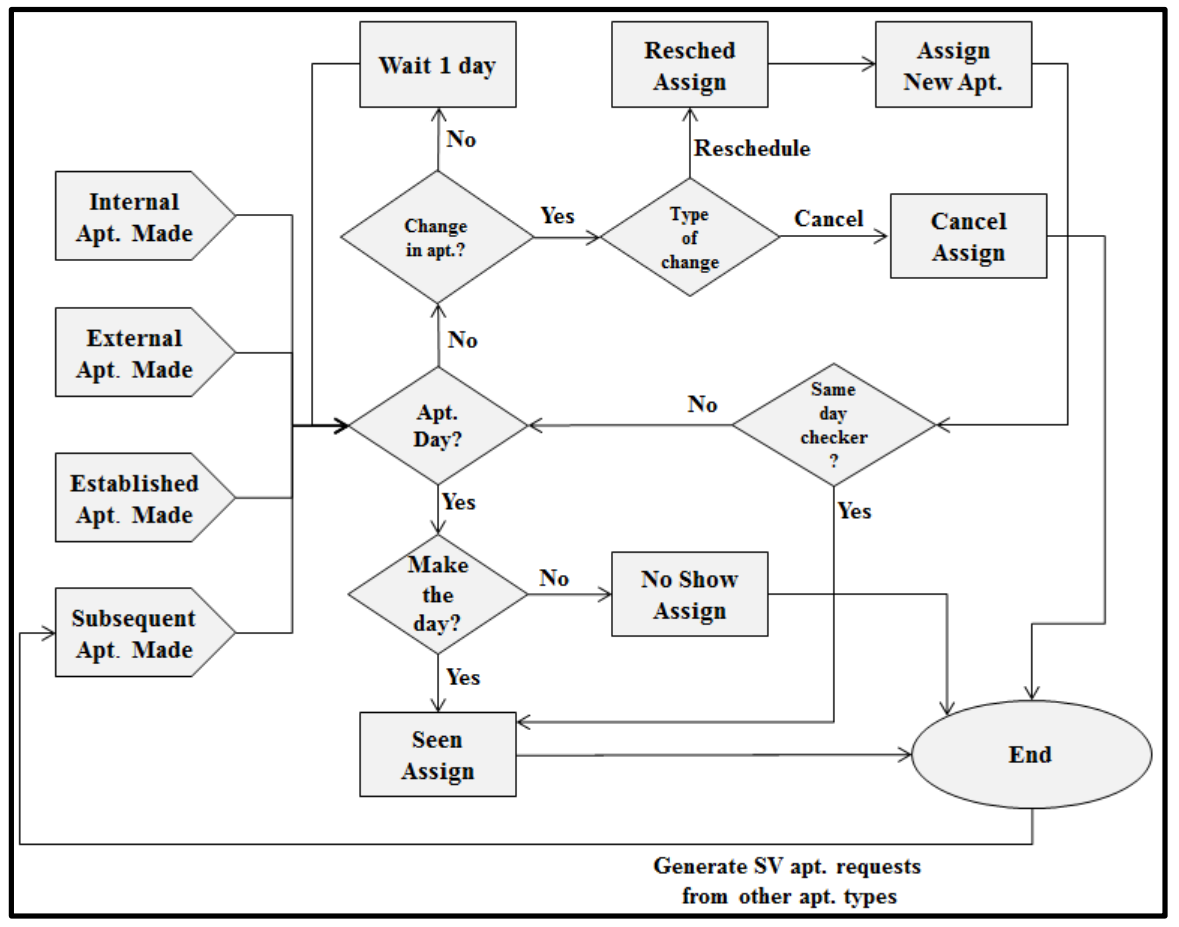

Figure 2: Process flow chart of the simulation model 
they are follow up visits of the other types. In this model, if an appointment is cancelled or rescheduled, that slot becomes available for other appointment requests, otherwise the patient will either be seen or will no-show based on behavioral functions of that class.

We first model the current scheduling system, in which the "current days out" functions are used to determine the assigned appointment days for incoming appointment requests, so that we can represent the current system with all its delays due to flaws in the capacity management. Appointment windows for all patient classes are 12-weeks in the current system, which we also refer to as the baseline model. We validate the model based on the days spent in the system by each patient class and the proportions of seen, no-show and cancelled patient appointments, and verify that these values are within $95 \%$ confidence interval of the historical data (Akin et al. 2013).

The simulation model is then modified to evaluate different scenarios for our experimental study. Under our proposed system we do not have predefined slots of different types. Instead we have generic 20 minutes length of slots to which any appointment can be assigned. Note that while assigning appointments, sufficient numbers of slots should be assigned to the patients based on their classes, i.e., two slots to an internal patient, three slots to a new external patient, one slot to subsequent visit patient, one, two or three slots to an established patient depending on the appointment type he/she needs. We use this model to evaluate various scenarios in which we use different appointment windows for different patient classes. While changing the appointment windows, we actually skew the current days out functions of each group by using multipliers in a way that the maximum appointment delays will be the target appointment windows for that scenario. Note that in order to prevent infeasible cases, if there is no available slot on the assigned day, the patient is moved to the next day until an available day is found.

In each scenario that is explained in the next subsection, daily net profit, seen patient percentages and overall utilization of the physicians are used as the performance indicators with $95 \%$ confidence intervals which are shown by black bars in the graphs in the results section. For each scenario, we use 1000 days to warm up the system, and once the system reaches the steady state we collect results for 260 weekdays (52 weeks).

\subsection{Scenarios}

In order to identify the scenario set for our experimental analysis, we use the choices shown in Table 1 for the appointment windows for each patient class. Note that, in all of the scenarios, we kept daily appointment requests of different patient classes, patient behavior functions, and daily resource capacities the same as they are in the current system (as explained in Section 3.1).

Table 1: Appointment window choices

\begin{tabular}{|l|l|l|}
\hline Patient Types & $\begin{array}{l}\text { Appointment Window } \\
\text { Choices }\end{array}$ & $\begin{array}{l}\text { Current System } \\
\text { Appointment Windows }\end{array}$ \\
\hline Internal Patients (INT) & 1 day, 2 days, 1 week & \multirow{2}{*}{ 12 weeks for all patient types } \\
\cline { 1 - 2 } New External Patients (EXT) & $1,3,6$ weeks & \\
\cline { 1 - 2 } Subsequent Visit Patients (SV) & $1,3,6$ weeks & \\
\cline { 1 - 2 } Established Patients (EST) & $6,9,12$ weeks & \\
\hline
\end{tabular}

When identifying the set of possible scenarios, we need to keep the appointment windows (AW) order between different patient classes as AW(INT) $\leq \mathrm{AW}(\mathrm{EXT}) \leq \mathrm{AW}(\mathrm{SV}) \leq \mathrm{AW}(\mathrm{EST})$ so that each scenario is accurate in terms of the patient admission priorities. The underlying logic for this order is that the clinic feels that because internal patients are already in the clinic they need to be seen as soon as possible. The main concern with the external patients is that they have not been seen previously in this clinic, and thus they do not have a commitment yet. For this reason they need to be seen quickly, but not necessary as early as the internal patients. For the subsequent visit patients, since they are usually following up 
on their previous visit, they need to be seen in a reasonable amount of time but this can be longer than the appointment windows for the external patients. Finally, established patients can be given the longest appointment window, as their appointment tends to be less urgent compared to the other types. Based on this constraint we identified 54 scenarios to test in addition to the current/baseline model in which 12 weeks appointment window is used for each patient class. A complete list of scenarios is given in Table 2.

\section{RESULTS}

For each scenario, the net profit (as a function of the relative value unit), the seen patient percentages, and the total utilization are shown in Figures $3 a, 3 b, 3 c$, respectively. The first observation that we can make with this experimental analysis is that, all of the proposed scenarios perform significantly better than the baseline in terms of all performance indicators with $95 \%$ confidence.

From Figure 3a, we can see that the best performing scenarios, based on Net Profit performance indicator, are scenarios $16,32,34$, and 53 in which we choose the shortest appointment window, i.e. 5 days, for the external patients. If we look at the seen patient percentages, there are other scenarios that perform close to these or better (see Figure 3b). However as we discuss in the following subsections the increase in overall seen patient percentages does not necessarily indicate that those scenarios perform well in terms of the net profit. Finally, in terms of utilization, all scenarios perform well, i.e. there is not a significant difference in this, between the scenarios (see Figure 3c). Next we will analyze the scenarios in subsets to see the effects of changing appointment windows for each patient type.

\subsection{Effects of the Internal Patients}

In order to see the effects of reducing the appointment window for the Internal Patients, we evaluate the change in the performance indicators within subsets of three (in the current order) in Figures $3 \mathrm{a}, 3 \mathrm{~b}$, and $3 \mathrm{c}$, starting with scenario 1 . In other words, by comparing scenarios 1,2 , and 3 , scenarios 4,5 , and 6 , and scenarios 7, 8, and 9, and so on, we can assess the change in the performance indicators as we set the appointment window for internal patients to 5,2 , and 1 days, respectively, while keeping the other parameters fixed.

This analysis suggests that reducing the appointment window for the internal patients does not always increase the daily net profit, indeed the net profit decreases in most of the scenario subsets as the appointment window is decreased from 2 days to 1 day (see Figure 3a). The reason of this reduction is that reducing the appointment window to 1 day, forces the model to give next day appointments to all internal patients and since we observe the same number of internal appointment requests each day, this creates a potential block in the system. Recall from Section 3 that for each patient type, after the initial appointment day is identified considering the appointment window, if that day is not available, the simulation schedules the patient for the first available day after that day. Thus, filling up the days with the internal patients prevents other patients from being given appointments in a timely manner. Additionally, as we know that the internal patients have the least revenue, filling the next day with them causes other patients, who have potentially higher revenue, to get later appointments (due to blocking) reduces the daily net profit. This is the reason of having higher seen patient percentages for some cases of 1 day versus 2 day appointment windows (see Figure 3b). In other words although in some cases we have higher seen patient percentages for the 1 day case, the net profit is not higher for those cases as we increase the internal patients in the daily mix and prevent other types from having timely access to the system. A comparison of the utilization in Figure $3 \mathrm{c}$ for these subsets shows a similar pattern. Although the difference is not significant, utilization decreases in most cases as we decrease the appointment window for the internal patients.

On the other hand, although it is not significant, we still need to note that for some cases reducing the appointment window from 5 days to 2 days improves the system in terms of daily net profit. The reason for this is that for those scenarios the capacity used for accommodating internal patients within two days is sufficient and does not cause blocking in the system, so that the other patients are not postponed further 
Akin, Ivy, Huschka, Rohleder, and Marmor

Table 2: Scenarios for appointment windows (in days)

\begin{tabular}{|c|c|c|c|c|c|c|c|c|c|c|c|c|c|c|}
\hline Scenario & INT & EXT & SV & EST & Scenario & INT & EXT & SV & EST & Scenario & INT & EXT & SV & EST \\
\hline $\mathbf{1}$ & 5 & 30 & 30 & 60 & $\mathbf{1 9}$ & 5 & 30 & 30 & 45 & $\mathbf{3 7}$ & 5 & 30 & 30 & 30 \\
\hline $\mathbf{2}$ & 2 & 30 & 30 & 60 & $\mathbf{2 0}$ & 2 & 30 & 30 & 45 & $\mathbf{3 8}$ & 2 & 30 & 30 & 30 \\
\hline $\mathbf{3}$ & 1 & 30 & 30 & 60 & $\mathbf{2 1}$ & 1 & 30 & 30 & 45 & $\mathbf{3 9}$ & 1 & 30 & 30 & 30 \\
\hline $\mathbf{4}$ & 5 & 15 & 30 & 60 & $\mathbf{2 2}$ & 5 & 15 & 30 & 45 & $\mathbf{4 0}$ & 5 & 15 & 30 & 30 \\
\hline $\mathbf{5}$ & 2 & 15 & 30 & 60 & $\mathbf{2 3}$ & 2 & 15 & 30 & 45 & $\mathbf{4 1}$ & 2 & 15 & 30 & 30 \\
\hline $\mathbf{6}$ & 1 & 15 & 30 & 60 & $\mathbf{2 4}$ & 1 & 15 & 30 & 45 & $\mathbf{4 2}$ & 1 & 15 & 30 & 30 \\
\hline $\mathbf{7}$ & 5 & 5 & 30 & 60 & $\mathbf{2 5}$ & 5 & 5 & 30 & 45 & $\mathbf{4 3}$ & 5 & 5 & 30 & 30 \\
\hline $\mathbf{8}$ & 2 & 5 & 30 & 60 & $\mathbf{2 6}$ & 2 & 5 & 30 & 45 & $\mathbf{4 4}$ & 2 & 5 & 30 & 30 \\
\hline $\mathbf{9}$ & 1 & 5 & 30 & 60 & $\mathbf{2 7}$ & 1 & 5 & 30 & 45 & $\mathbf{4 5}$ & 1 & 5 & 30 & 30 \\
\hline $\mathbf{1 0}$ & 5 & 15 & 15 & 60 & $\mathbf{2 8}$ & 5 & 15 & 15 & 45 & $\mathbf{4 6}$ & 5 & 15 & 15 & 30 \\
\hline $\mathbf{1 1}$ & 2 & 15 & 15 & 60 & $\mathbf{2 9}$ & 2 & 15 & 15 & 45 & $\mathbf{4 7}$ & 2 & 15 & 15 & 30 \\
\hline $\mathbf{1 2}$ & 1 & 15 & 15 & 60 & $\mathbf{3 0}$ & 1 & 15 & 15 & 45 & $\mathbf{4 8}$ & 1 & 15 & 15 & 30 \\
\hline $\mathbf{1 3}$ & 5 & 5 & 15 & 60 & $\mathbf{3 1}$ & 5 & 5 & 15 & 45 & $\mathbf{4 9}$ & 5 & 5 & 15 & 30 \\
\hline $\mathbf{1 4}$ & 2 & 5 & 15 & 60 & $\mathbf{3 2}$ & 2 & 5 & 15 & 45 & $\mathbf{5 0}$ & 2 & 5 & 15 & 30 \\
\hline $\mathbf{1 5}$ & 1 & 5 & 15 & 60 & $\mathbf{3 3}$ & 1 & 5 & 15 & 45 & $\mathbf{5 1}$ & 1 & 5 & 15 & 30 \\
\hline $\mathbf{1 6}$ & 5 & 5 & 5 & 60 & $\mathbf{3 4}$ & 5 & 5 & 5 & 45 & $\mathbf{5 2}$ & 5 & 5 & 5 & 30 \\
\hline $\mathbf{1 7}$ & 2 & 5 & 5 & 60 & $\mathbf{3 5}$ & 2 & 5 & 5 & 45 & $\mathbf{5 3}$ & 2 & 5 & 5 & 30 \\
\hline $\mathbf{1 8}$ & 1 & 5 & 5 & 60 & $\mathbf{3 6}$ & 1 & 5 & 5 & 45 & $\mathbf{5 4}$ & 1 & 5 & 5 & 30 \\
\hline
\end{tabular}
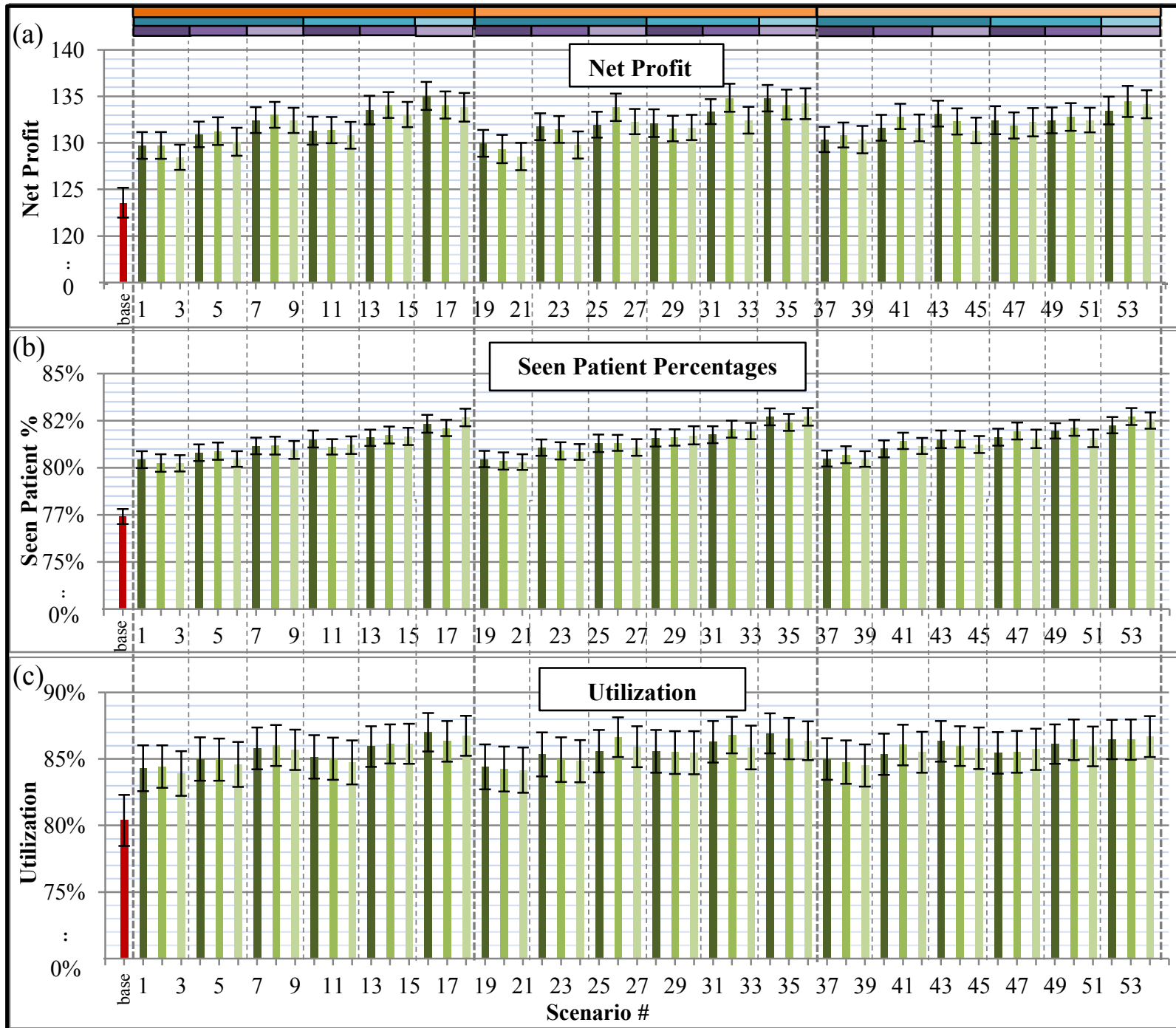

Figure 3: (a) Net profit per day, (b) Percentage of the seen patients per day, (c) Total utilization per day 
than their initially identified appointment days. Thus, the 2-day case obtains higher profit compared to 5day case by seeing more internal patients without blocking, and the profit is not significantly different for seeing other types of patients in both cases. This suggests that we can increase the daily net profit by choosing a 2-day instead of a 5-day appointment window for those settings.

Finally, we note one limitation of our model is that the net profit associated with an internal patient is more complex than we have been able to incorporate. Internal patients feed from other departments, they are likely to have a relative value unit somewhat higher to the clinic as a whole than the value within this specific department.

\subsection{Effects of the New External Patients}

In order to evaluate the effect of decreasing the appointment window for the new external patients, we compare the scenarios within subsets of 2-3 scenarios, depending on the available appointment window choices for the new external patients for those subsets, e.g., $\{29,14,4\}$ or $\{14,4\}$ respectively, since we have to satisfy $\mathrm{AW}(\mathrm{INT}) \leq \mathrm{AW}(\mathrm{EXT}) \leq \mathrm{AW}(\mathrm{SV}) \leq \mathrm{AW}(\mathrm{EST})$ relationship between the appointment windows of different patient types. Thus, we evaluate the following subsets of scenarios: $\{1,4,7\},\{2,5$, $8\},\{3,6,9\},\{10,13\},\{11,14\},\{12,15\},\{19,22,25\},\{20,23,26\},\{21,24,27\},\{28,31\},\{29,32\}$, $\{30,33\},\{37,40,43\},\{38,41,44\},\{39,42,45\},\{46,49\},\{47,50\}$, and $\{48,51\}$.

After comparing the scenarios within the above subsets, we observe that reducing the appointment window for the new external patients increases the net profit significantly in most cases, along with the seen patient percentages and utilization, although the improvements in the latter two indicators are not significant. Unlike the internal patients, the new external patients have the highest revenue, thus although reducing their appointment window may cause blocking for other types of patients, the revenue gained by giving these patients priority is still worthwhile in terms of the net profit. However, the significance of the profit increase, reduces for the latter subsets of scenarios. The reason for this reduction in the significance is that, for the latter scenarios all patient types have shorter appointment windows and this causes the earlier days to fill up rapidly, and the system pushes the new appointments to later days until it finds an available day. Similar to our discussion in the previous subsection for the internal patients, this might create blocking in the system and this is why for those latter scenario subsets which reduce new external patients even more, do not improve the system significantly.

\subsection{Effects of the Subsequent Visit Patients}

Subsequent visit patients also have higher revenues ( 2 units) compared to the established ( 1 unit) and internal patients ( 0.8 unit), and close to new external patients ( 3 units). Thus we can expect to have similar results to these for the new external patients, but the significance in the improvement is expected to be a bit lower compared to the external patients. Because as shown in the Figure 1, external patients have a steeper decrease in their seen probabilities as the appointment delay increases, compared to subsequent visit patients. In order to assess the effects of decreasing the appointment window for the subsequent visit patient, we compare the following subsets of scenarios: $\{4,10\},\{5,11\},\{6,12\},\{7,13,16\},\{8,14$, $17\},\{9,15,18\},\{22,28\},\{23,29\},\{24,30\},\{25,31,34\},\{26,32,35\},\{27,33,36\},\{40,46\},\{41$, $47\},\{42,48\},\{43,49,52\},\{44,50,53\},\{45,51,54\}$. As shown in Figure 3a, the net profit increases significantly with $95 \%$ confidence in most cases, as the appointment window decreases for subsequent visit patients. The seen patient percentages and the utilization also have the similar pattern, although the change in the utilization is not significant within $95 \%$ confidence (see Figures $3 \mathrm{~b}$ and $3 \mathrm{c}$ ). Note that there are only a few subsets in which the changes in the performance indicators are very low, for the reasons as discussed previously. 


\subsection{Effects of the Established Patients}

Finally, in order to assess the effect of decreasing the appointment window for established patients we compare the scenarios within the following subsets: $\{1,19,37\},\{2,20,38\},\{3,21,39\},\{4,22,40\},\{5$, $23,41\},\{6,24,42\},\{7,25,43\},\{8,26,44\},\{9,27,45\},\{10,28,46\},\{11,29,47\},\{12,30,48\},\{13$, $31,49\},\{14,32,50\},\{15,33,51\},\{16,34,52\},\{17,35,53\}$, and $\{18,36,54\}$.

In this case, we cannot directly conclude that the performance indicators always increase or decrease as we reduce the appointment window. This can be explained with the fact that the established patient type has lower revenue compared to the new external and subsequent visit patients, but higher compared to the internal patients. Moreover, when we look at the behavioral functions for this patient type, we observe that the probability of being seen curve is flatter than all other patient types. For this reason, reducing appointment window for this patient class does not necessarily increase the net profit, as in some cases it can cause blocking in the system and prevents other patients from being admitted in a timely manner, and reduces the net profit. What we observe from Figures $3 a, 3 b$, and $3 c$ for the scenario subsets in which the appointment window for external patients is 5 days, reducing the appointment window for established patients actually reduces the net profit, although it is not significant. By having appointment windows for the external patients at the lowest value, the early days fill up rapidly and if we also want to reduce the established patients' appointment window, this creates blocking in the system due to capacity restrictions, so that the patients with higher revenues (compared to established patients) are delayed until an available slot is found. For the other scenarios there is not a significant increase in the performance indicators. Thus, given the lower revenue and the flatter seen probability curve, it is not worthwhile to reduce the appointment window for this patient type.

\section{CONCLUSION}

In this paper, we consider an outpatient clinic with multiple physicians and multiple patients each of which has different revenue, different appointment request rate, different service time and different behavioral functions (cancellation, reschedule, no-show, seen) that are appointment delay dependent. Under this setting, we evaluate the effects of changing appointment windows for different patient types with the performance indicators of net profit, seen patient percentages and overall utilization of physicians. Due to the complexity of the system we use simulation with Arena in order to understand the system and explore the advantages and disadvantages of using different and shorter appointment windows for each patient class.

Based on our results we conclude that it is possible to significantly improve all of our performance indicators by using the scenarios, in which the largest appointment window is 1 week for internal patients (Scenario 2), 6 weeks for new external patients, 6 weeks for subsequent visit patients, and 12 weeks for established patients. In other words, by making only these changes in the current system we can improve the clinic performance indicators significantly. However, although all scenarios are better than the current system, if we compare the proposed scenarios by each other, we cannot directly conclude that reducing appointment windows always increases the performance indicators. For example, for the internal patients reducing the appointment windows to 1 day is actually worse than 2 days. We can explain this with the fact that filling the next day with the internal patients would cause other patients to not be able to enter the system in a timely manner, and given the lower revenue obtained from internal patients, it is not worthwhile to give them next day appointments. However, with the new external patients we observe an increase in the net profit in most cases as we reduce the appointment window, this is due to high revenue that they bring into the clinic and compensates for delaying patients of other types. For this reason, it is important for the clinic to prioritize their performance indicators while considering the trade-off between scheduling a patient of a certain class to an earlier versus a later day by taking their blocking effect on the system into account.

An interesting area for future research is to incorporate the fact that established patients are generated by new external patients and internal patients. In this paper we are not able to explore the relationship be- 
tween established patients and new external patients and internal patients due to data limitations. However, by defining established patients as functions of other patient types we can explore the effect of the dependency when increasing the load on the system.

\section{REFERENCES}

Akin, G., Ivy, J., Huschka, T. R., Rohleder, T. R., 2013, "Simulation-Based Analysis of Scheduling Decisions in an Outpatient Clinic," Proceedings of the 2013 ISERC.

Ayvaz, N., Huh, W., 2010, "Allocation of Hospital Capacity to Multiple Types of Patients," Journal of Revenue \& Pricing Management, 9(5), 386-398.

Cayirli, T., Veral, E., 2003, "Outpatient Scheduling in Health Care: A Review of Literature," Production and Operations Management, 12(4), 519-549.

Cayirli, T., Veral, E., Rosen, H., 2008, "Assessment of Patient Classification in Appointment System Design," Production and Operations Management, 17(3), 338-353.

Chakraborty, S., Muthuraman, K., Lawley, M., 2010, "Sequential Clinical Scheduling with Patient NoShows and General Service Time Distributions," IIE Transactions, 42(5), 354-366.

Feldman, J., Liu, N., Topaloglu, H., Ziya, S., 2012, "Appointment Scheduling under Patient Preference and No-Show Behavior," Working Paper.

Gallucci, G., Swartz, W., Hackerman, F., 2005, "Brief Reports: Impact of the Wait for an Initial Appointment on the Rate of Kept Appointments at a Mental Health Center," Psychiatr.Serv.,56(3), 344346.

Green, L. V., Savin, S., 2008, “Reducing Delays for Medical Appointments: A Queueing Approach,” Operations Research, 56(6), 1526-1538.

Gupta, D., Denton, B., 2008, "Appointment Scheduling in Health Care: Challenges and Opportunities," IIE Transactions, 40(9), 800-819.

Gupta, D., Wang, L., 2008, "Revenue management for a primary-care clinic in the presence of patient choice," Operations Research, 56(3), 576-592.

Huh, W. T., Liu, N., Truong, V. A., 2013, "Multi-resource Allocation Scheduling in Dynamic Environments," MSOM, in press.

LaGanga, L. R., Lawrence, S. R., 2007, "Clinic Overbooking to Improve Patient Access and Increase Provider Productivity," Decision Sciences, 38(2), 251-276.

Liu, N., Ziya, S., Kulkarni, V. G., 2010, "Dynamic Scheduling of Outpatient Appointments Under Patient No-Shows and Cancellations," MSOM, 12(2), 347-364.

Muthuraman, K., Lawley, M., 2008, "A Stochastic Overbooking Model for Outpatient Clinical Scheduling with No-Shows", IIE Transactions, 40(9), 820-837.

Patrick, J., Puterman, M. L, Queyranne, M., 2008, "Dynamic Multi-Priority Patient Scheduling for a Diagnostic Resource," Operations Research, 56(6), 1507-1525.

Patrick, J., 2012, “A Markov Decision Model for Determining Optimal Outpatient Scheduling," Health Care Management Science, 15, 91-102.

Ratcliffe, A., Gilland, W., Marucheck, A., 2012, "Revenue Management for Outpatient Appointments: Joint Capacity Control and Overbooking with Class-Dependent No-shows," Flex Serv Manuf J., 24(4), 516-548.

Samorani, M., LaGanga, L., 2011, "Scheduling Appointments in a Multi-Day Scheduling Horizon Given Individual Show Probabilities," Working Paper.

Shutz, H., Kolisch, R., 2013, "Capacity Allocation for Demand of Different Customer Product Combinations with Cancellations, No Shows, and Overbooking When There is a Sequential Delivery of Service," Annals of Operations Research, 206(1), 401-423.

White, D. L., Froehle, C. M., Klassen, K. J., 2011, "The Effect of Integrated Scheduling and Capacity Policies on Clinical Efficiency," Production and Operations Management, 20(3), 442-455. 
Zeng, B., Turkcan, A., Lin, J., Lawley, M., 2010, "Clinic Scheduling Models with Overbooking for Patients with Heterogeneous No-Show Probabilities," Annals of Operations Research, 178(1), 121- 144.

\section{AUTHOR BIOGRAPHIES}

GOKCE AKIN is a Ph.D. student in Operations Research at North Carolina State University. She received her BS degree in Industrial Engineering in 2008 and MS degree in Industrial Engineering in 2010 at Bilkent University in Ankara, Turkey. Her research interests include computer simulation, capacity and revenue management, inventory control, and optimization in healthcare. Her email address is gakin@ncsu.edu.

JULIE S. IVY is an Associate Professor in the Edward P. Fitts Department of Industrial and Systems Engineering at North Carolina State University. Her primary research interests are in the mathematical modeling of stochastic dynamic systems with emphasis on statistics and decision analysis as applied to health care, manufacturing, and service environments. The focus of her research is decision making under conditions of uncertainty with the objective of improving the decision quality. Her email address is jsivy@ncsu.edu.

TODD R. HUSCHKA is a Master Health Systems Analyst in the Division of Health Care Policy and Research at Mayo Clinic. He has been at the Mayo Clinic for 15 years, with the past 6 years focusing on Operations Research. He has a MS in Industrial Engineering from the University of Wisconsin, and a BS in Statistics from North Dakota State University. His primary focus of research has been in the simulation/optimization of surgical and clinical schedules as well as the optimization of hospital resources. He has several publications and has been regular presenter at both INFORMS and WSC. His email address is huschka.todd@mayo.edu.

THOMAS R. ROHLEDER is a Professor of Health Care Systems Engineering in the Division of Health Care Policy and Research at Mayo Clinic. He was as previously a professor of Operations Management in the Haskayne School of Business at the University of Calgary. He has a Ph.D. in Business Administration, and B.S.B. in Finance from the University of Minnesota. Before obtaining his doctorate, Dr. Rohleder worked in the financial services sector for ITT Commercial Finance Corp., and Northwestern National Life Insurance Co. Dr. Rohleder has over 40 publications in journals such as the Journal of Operations Management, Production and Operations Management, and Health Care Management Science. He is currently working on research in the areas of health systems engineering and health care operations management. His email address is rohleder@mayo.edu.

YARIV N. MARMOR is an Assistant Professor of Health Care Systems Engineering in the Division of Health Care Policy and Research at Mayo Clinic. He holds a PhD in Industrial Engineering from the Technion-Israel Institute of Technology. His current research interests are in the area of process analysis and improvement of medical systems. Dr. Marmor has published his research in journals such as ACM Transactions on Modeling and Computer Simulation (TOMACS), International Journal of Production Research, IIE Transactions, and Journal of Health Organization and Management. His email address is marmor.yariv@mayo.edu. 